\title{
NO-synthase activity in mitochondria of uterus smooth muscle: identification and biochemical properties
}

\author{
Hanna V. Danylovych, Yuriy V. Danylovych, Maria O. Gulina, Tatyana V. Bohach \\ and Sergiy O. Kosterin
}

Palladin Institute of Biochemistry of NAS of Ukraine, Kyiv, Ukraine

\begin{abstract}
Information about the catalytic and kinetic properties of mitochondria NO-synthase from uterus smooth muscle is missing currently. According to the data on MitoTracker Orange CMH2TMRos and 4-amino-5-methylamino-2',7'-difluorescein, diaminofluorescein-FM (DAF-FM) dye co-localization in uterine smooth muscle cells, presented in this paper, NO can be synthesized in their mitochondria. High activity of NO synthase requires the presence of substrates of respiration, $\mathrm{L}$-arginine, $\mathrm{Ca}^{2+}$ and NADPH. It is established that the dependence of NO production on the concentration of L-arginine has a bell-shaped character with a maximum of $75 \mu \mathrm{M}$, and the apparent affinity constant for L-arginine is $28.9 \pm 9.1 \mu \mathrm{M}$. The dependence of $\mathrm{NO}$ production on $\mathrm{Ca}^{2+}$ concentration has a maximum at $100-250 \mu \mathrm{M}$; the activation constant for $\mathrm{Ca}^{2+}$ is $44.4 \pm 14.5 \mu \mathrm{M}$. The inhibitor of $\mathrm{Ca}^{2+}$ transport in mitochondria ruthenium red (RuR), as well as the inhibitor of NO-synthase $\mathrm{N}^{\mathrm{G}}$-nitro-L-arginine (NA), reduces $\mathrm{NO}$ production. The biosynthesis of NO by mitochondria depends on its energized level: it is stimulated by the addition of respiration substrates, suppressed with specific inhibitors of the electron transport chain (rotenone and antimycin A) and carbonyl-cyanide 3-chlorophenylhydrazone (CCCP) protonophore.
\end{abstract}

Key words: Nitric oxide - Mitochondria - Calcium - Smooth muscle - Myometrium

\section{Introduction}

Nitric oxide (NO) is a universal signaling and regulatory molecule in a cell. The control of the contractile function of smooth muscles, including myometrium, is an important example of its biological activity (Buxton 2004).

To date, it has been found that NO modulates energy, metabolic and transport processes in mitochondria. It is a well-known point of view that mitochondria are the primary cell targets of NO (Giulivi 2007). The membrane of mitochondria is an effective target of nitrocompounds, since there is a high content of thiol residues, iron-sulfur centers, hemic groups in the localized enzymes, and the membrane itself is the place of superoxide anion formation (Boveris 1977; Korshunov et al. 1997; Ghimire 2017). Nitric oxide is capable of regulating the activity of the mitochondrial electron transport chain, reversibly suppressing

Correspondence to: Hanna V. Danylovych, Palladin Institute of Biochemistry of NAS of Ukraine, Leontovicha 9, 01030 Kyiv, Ukraine E-mail: danylovych@biochem.kiev.ua cytochrome c-oxidase, and controlling mitochondrial $\mathrm{pH}$ (Giulivi 2007; Piantadosi and Suliman 2012; Ghimire 2017). At low nanomolar concentrations, NO limits the intensity of respiration and oxidative phosphorylation, which is considered as an adaptive physiological response (Brown 2007; Brown and Borutaite 2007; Levine et al. 2012; Zaobornyj and Ghafourifar 2012). NO regulates the homeostasis of $\mathrm{Ca}^{2+}$ in mitochondria and, accordingly, $\mathrm{Ca}^{2+}$-dependent processes, primarily the activity of dehydrogenases (Traaseth et al. 2004; Giulivi et al. 2006; Gellerich et al. 2010). NO/cGMPdependent decrease in the electrical potential on the internal membrane of mitochondria, which affects the intensity of electrophoretic accumulation of $\mathrm{Ca}^{2+}$, is described (Levine et al. 2012). The synthesis of ATP by mitochondria is regulated by NO in a GMP-dependent manner (Forstermann and Sessa 2012; Levine et al. 2012). Nitric oxide stimulates mitochondria biogenesis in various types of tissues and, in particular, increases the expression of sirtuin-1, which together with 5'AMP protein kinase regulates peroxisome proliferator-activated receptor- $\gamma$ coactivator-1 $\alpha$ (PGC-1 $\alpha$ ), which is a modulator of mitochondrial biogenesis (Nisoli and 
Carruba 2006; Levine et al. 2012; Piantadosi and Suliman 2012; Tengan et al. 2012). There is no doubt that biochemistry and molecular physiology of mitochondria, which combines the processes of oxygen consumption, oxidative phosphorylation, lipolytic catabolism, heme biosynthesis, $\mathrm{Ca}^{2+}$ homeostasis maintenance, oxygen active form production and apoptosis, are largely controlled by NO (Giulivi et al. 2006).

Considering the central role of mitochondria in cellular energy supply, and also taking into account modern notions of mitochondria as a key element in biochemical processes of endothelial dysfunction in cardiovascular diseases, type II diabetes, neurodegenerative pathologies etc. ("mitochondrial diseases"), considerable attention in modern literature is given to the problem of the relationship: $\mathrm{NO}$ - mitochondria (Davidson and Duchen 2006; Levine et al. 2012; Litvinova et al. 2015).

In 1997 P. Ghafourifar and C. Richter demonstrated the existence of functionally active mitochondrial NOS (common abbreviation - mtNOS) in rat liver mitochondria. The enzyme formed NO and L-citrulline in the presence of L-arginine, and the process was $\mathrm{Ca}^{2+}$-dependent. Such analogues of L-arginine as $\mathrm{N}^{\mathrm{G}}$-monomethyl-L-arginine (L-NMMA) and $\mathrm{N}^{\mathrm{G}}$-nitro-L-arginine (NA) inhibited the enzyme. The activity of mtNOS was associated with the internal mitochondrial membrane. The enzyme was constitutively active, dependent on the intensity of mitochondrial respiration and was under the control of membrane potential in intact energized by succinate addition mitochondria (Ghafourifar and Richter 1997). In 1998 C. Giulivi and A. Boveris with co-authors (Giulivi et al. 1998) showed NO production in rat liver mitochondria by direct, reliable, precise independent methods: electron-paramagnetic resonance using spin traps (derivatives of dithiocarbamates) and two-wavelength spectrophotometry of oxidized hemoglobin. Studies were conducted on purified preparations of mitochondria (intact, permeabilized organelles, sub-mitochondrial particles) and crude fraction of mtNOS. The apparent $\mathrm{K}_{\mathrm{m}}$ for L-arginine was also determined, which was $5-7 \mu \mathrm{M}$, being close to that for neuronal NO-synthase (nNOS) (Haynes et al. 2004; Valdez et al. 2006).

The majority of experimental data suggests that in mitochondria a special isoform of NOS exists - mtNOS, with a molecular weight of $144 \mathrm{kDa}$, which is a splice variant of nNOS- $\alpha$ different from those already identified. It has two post-translational modifications: $\mathrm{N}$-terminal myristoylation on Thr or Ser residues and C-terminal phosphorylation on Ser-1412 (Elfering et al. 2002; Haynes et al. 2004; Traaseth et al. 2004; Ghafourifar and Cadenas 2005). Immune co-localization of mtNOS with mitochondrial markers (succinate dehydrogenase, cytochrome c-oxidase) further proves its placement precisely in the internal mitochondrial membrane. mtNOS myristoylation is necessary to direct the enzyme to a mitochondria or to anchor it in the mem- brane. Phosphorylation of C-terminal Ser residue regulates the activity of mtNOS (Elfering et al. 2002; Zaobornyj and Ghafourifar 2012; Tengan et al. 2012).

Immune-electron microscopy and immune-precipitation techniques show the interaction of mtNOS with the subunit $\mathrm{V}_{\mathrm{a}}$ of cytochrome c-oxidase via the PDZ domain (acronym from words: post, Drosophila, Zonula). Other studies have found that the proteins of complex I of the electron transport chain are immune-precipitated with intra-mitochondrial NOS (Ghafourifar and Cadenas 2005; Persichini et al. 2005; Franco et al. 2006; Carreras and Poderoso 2007; Zaobornyj and Ghafourifar 2012).

mtNOS-generated NO provides reciprocal regulation between the biosynthesis of NO in this compartment and the concentration of intra mitochondrial $\mathrm{Ca}^{2+}$, L-arginine, $\mathrm{pH}, \mathrm{O}_{2}$ partial pressure and oxidation-reduction state of mitochondria (Nisoli and Carruba 2006; Dedkova and Blatter 2009; Santos et al. 2011; Zaobornyj and Ghafourifar 2012). Localization of NOS in mitochondria is today reliably shown in various organs and tissues of mammals: liver (Giulivi 1998; Tatoyan and Giulivi 1998), heart (Davidson and Duchen 2006; Dedkova and Blatter 2009; Litvinova et al. 2015), brain (Dolińska and Albrecht 1998), diafragm (Haynes et al. 2003; Alvarez and Boveris 2004), kidneys (Boveris 2003), thymus (Bustamante et al. 2002). The biosynthesis of NO by mitochondria is a powerful process: heart and diaphragm organelles are capable to produce $67 \%$ and $24 \%$ of the total cell content of NO, respectively.

However, the possibility and the biochemical properties of $\mathrm{NO}$ formation in smooth muscle mitochondria remain to be clarified, and the problem of NO biosynthesis in myometrium mitochondria is not considered. The presented work is dedicated to the solution of these issues.

\section{Materials and Methods}

\section{Isolation of myocytes from the uterine smooth muscle (myometrium) of non-pregnant rats}

Rats were anesthetized by diethyl ether inhalation and decapitated. Myocytes were isolated from the uteruses of white nonlinear nonpregnant rats, by applying Mollard method using collagenase and soybean trypsin inhibitor (Mollard et al. 1986).

\section{Isolation of mitochondria from the uterine smooth muscle}

The mitochondrial fraction was isolated from the myometrium of non-pregnant rats using differential centrifugation, as described by Kosterin and coworkers (Kolomiets et al. 1985). Rats were anesthetized by diethyl ether inhalation and then decapitated. The isolated mitochondrial fraction was kept on ice for the duration of the experiment. The protein 
content in the mitochondrial fraction was determined by a standard procedure by Bradford (1976). The total protein content in the mitochondrial fraction was $2 \mathrm{mg} / \mathrm{ml}$.

Animal experiments were conducted in accordance with the guidelines of the European Convention for the Protection of Vertebrate Animals used for Experimental and Other Scientific Purposes (International Convention, Strasbourg, 1986).

\section{Laser scanning confocal microscopy}

Spatial distribution of fluorescent dyes in cells was investigated with the confocal laser scanning microscope LSM 510 META ( (Carl Zeiss», Germany). Myocytes were immobilized on poly-L-lysine. All experimental procedures were conducted in physiological Henks's solution $(\mathrm{mM}): \mathrm{NaCl}, 136.9 ; \mathrm{KCl}, 5.36 ; \mathrm{KH}_{2} \mathrm{PO}_{4}, 0.44 ; \mathrm{NaHCO}_{3}$, $0.26 ; \mathrm{Na}_{2} \mathrm{HPO}_{4}, 0.26 ; \mathrm{CaCl}_{2}, 0.03 ; \mathrm{MgCl}_{2}, 0.4 ; \mathrm{MgSO}_{4}$, 0.4 ; glucose, 5.5; Hepes $\left(\mathrm{pH} 7.4 ; 37^{\circ} \mathrm{C}\right), 10$. For the visualization of mitochondria and cell nucleus the fluorescent dyes MitoTracker Orange $\mathrm{CMH}_{2}$ TMRos (200 nM) and Hoechst 33342 (50 nM) were used, respectively (Buckman et al. 2001). The loading of immobilized myocytes with NO-sensitive fluorescent probe 4-amino-5-methylamino2',7'-difluorescein, diaminofluorescein-FM (DAF-FM) in concentration of $10 \mu \mathrm{M}$ was carried out for $15 \mathrm{~min}$ at $24^{\circ} \mathrm{C}$. The experiments with confocal microscopy were performed in MultiTrack mode. The fluorescence of Hoechst 33342 was excited using excitation laser at wavelength $405 \mathrm{~nm}$, and registered with the BP 420-480 filter. The excitation laser at the wavelength of $543 \mathrm{~nm}$ was used for MitoTracker Orange CM-H2TMRos, and the fluorescence was registered in the spectral range 560-615 nm; DAF-FM fluorescence was excited at the wavelength $488 \mathrm{~nm}$, and the emission was registered in the spectral range 505-530 $\mathrm{nm}$.

\section{Study of NO biosynthesis by isolated mitochondria using} DAF-FM and flow cytometry

The measurements were performed using COULTER EPICS XL ${ }^{\mathrm{TM}}$ (Beckman Coulter, USA) cytometer with an argon laser $\left(\lambda_{\mathrm{ex}}=488 \mathrm{~nm}, \lambda_{\mathrm{fl}}=515 \mathrm{~nm}\right.$ (Fl1 channel). The NO-sensitive DAF-FM fluorescence probe was added to the mitochondria in the working medium, the experimentallyselected concentration was $0.5 \mu \mathrm{M}$. In experiments DAF-FM was used at concentrations of $0.1-10 \mu \mathrm{M}$ to find optimal conditions for the determination of NO.

The dependence of mtNOS activity on the presence of respiratory substrates ( $5 \mathrm{mM}$ pyruvate and succinate), L-arginine $(1-100 \mu \mathrm{M}), \mathrm{Ca}^{2+}(10-500 \mu \mathrm{M}$, in some cases $1 \mathrm{mM})$, cofactors $\left(10 \mu \mathrm{M}\right.$ tetrahydrobiopterin $\left(\mathrm{BH}_{4}\right)$ and FAD), NADPH $(10 \mu \mathrm{M})$, as well as $10 \mu \mathrm{M}$ ruthenium red (RuR), $7.5 \mu \mathrm{g} / \mathrm{ml}$ alamethicin and $\mathrm{N}^{\mathrm{G}}$-nitro-L-arginine
(NA), was studied. ADP (0.5 mM), inhibitors of the electron transport chain $(5 \mu \mathrm{M}$ rotenone, $1 \mu \mathrm{g} / \mathrm{ml}$ antimycin $\mathrm{A}, 2.5$ $\mu \mathrm{M}$ oligomycin) and $10 \mu \mathrm{M}$ protonophore carbonyl-cyanide 3-chlorophenylhydrazone (CCCP) were used in the investigation of mitochondria NO production depending on their energy status.

The working medium in all cases, except for the concentration dependences on $\mathrm{L}$-arginine and $\mathrm{Ca}^{2+}$, was com-

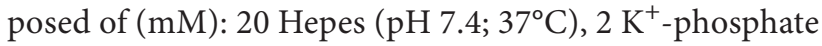
buffer $\left(\mathrm{pH} 7.4,37^{\circ} \mathrm{C}\right), 120 \mathrm{KCl}, 5$ pyruvate, 5 succinate, $0.05 \mathrm{~L}$-arginine, $0.1 \mathrm{Ca}^{2+}, 0.01 \mathrm{NADPH}, 0.01 \mathrm{BH}_{4}$, aliquot of the mitochondria fraction contained $20 \mu \mathrm{g}$ of protein.

The activation constant of NO-synthase reaction in $\mathrm{mi}-$ tochondria for $\mathrm{Ca}^{2+}$ and the apparent affinity constant for L-arginine were evaluated by Heinz method (Keleti 1986). These constants were calculated in Heinz coordinates $\{(\mathrm{S}) / \mathrm{V}$ : $(\mathrm{S})\}$, where (S), L-arginine or $\mathrm{Ca}^{2+}$ concentration; V, mtNOS activity $(n=5)$. Correlation coefficient formulas are used to find how strong a relationship between the data is. The formulas return a value between -1 and 1 . Curves with the correlation coefficient $\mathrm{R}^{2}>0.9$ were taken into account. The data are presented as mean \pm SE.

In the work the following reagents were used: Hepes, glucose, saccharose, sodium succinate, sodium pyruvate, bovine serum albumin, poly-L-lysine, collagenase type IA, ATP, ADP, Pluronic F-27, DAF-FM $\left(\mathrm{C}_{21} \mathrm{H}_{14} \mathrm{~F}_{2} \mathrm{~N}_{2} \mathrm{O}_{5}\right.$, 4-amino-5methylamino-2',7'-difluorescein, diaminofluorescein-FM), EGTA, $\mathrm{CaCl}_{2}, \mathrm{~N}^{\mathrm{G}}$-nitro-L-arginine, oligomycin, rotenone, antimycin A, CCCP, ruthenium red, L-arginine, NADPH, $\mathrm{BH}_{4}, \mathrm{FAD}$ (Sigma, USA); Hoechst 33342, soybean trypsin inhibitor (Fluka, Switzerland); MitoTracker Orange CMH2TMRos (Invitrogen, USA). Any other reagents are produced in Ukraine.

\section{Results}

We used NO-sensitive fluorescent probe DAF-FM, an active form of dye that enters the cell through simple diffusion, in order to prove the presence of $\mathrm{NO}$ in mitochondria. DAF-FM reacts with $\mathrm{NO}$ in the presence of $\mathrm{O}_{2}$, resulting in its triazolofluorescein derivative, which has a higher fluorescence efficiency in the green region (Li and Wan 2015). While using MitoTracker Orange and DAF-FM fluorescence dyes, their co-localization was observed (Fig. 1), proving the similar distribution of fluorescence probes in the cytoplasm. This indicates the presence of NO in mitochondria, which is consistent with the notion of the presence of mitochondrial $\mathrm{NO}$-synthase in these organelles.

Further research was aimed at the selection of optimal conditions for the determination of $\mathrm{NO}$-synthase activity in isolated mitochondria of rat myometrium using DAFFM and flow cytometry. The analysis of the fluorescence 

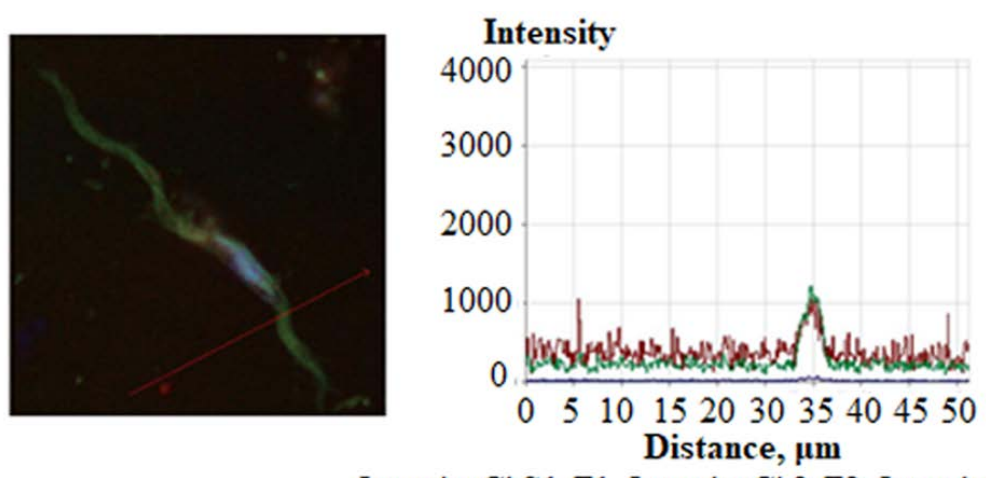

Intensity $\mathrm{ChS} 1-\mathrm{T} 1$ Intensity $\mathrm{Ch} 2-\mathrm{T} 2$ Intensity $\mathrm{Ch} 2-\mathrm{T} 3$
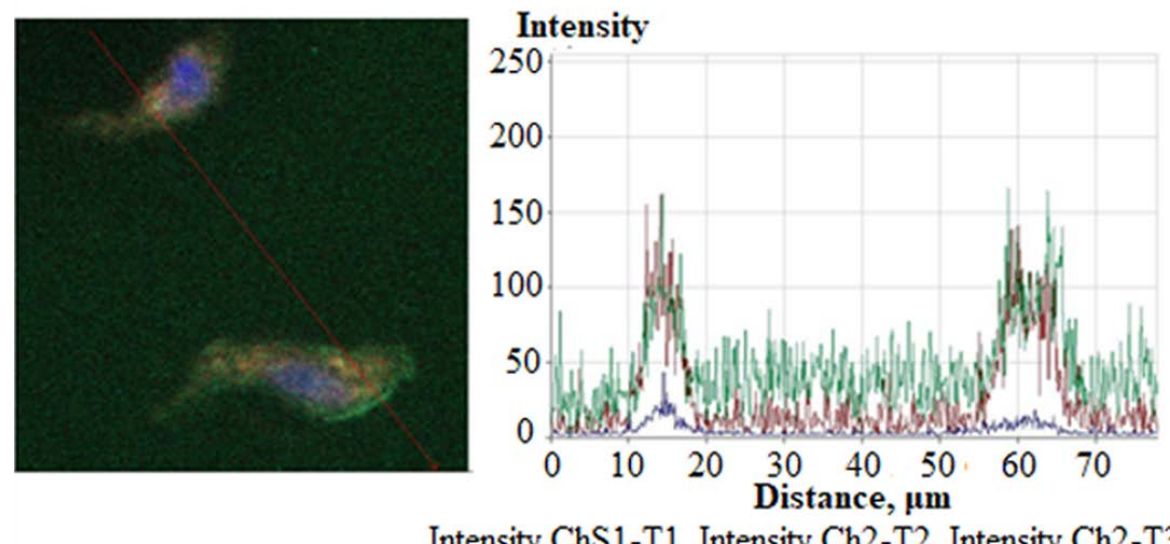

Figure 1. Co-localization of DAFFM and MitoTracker Orange CM$\mathrm{H}_{2}$ TMRos fluorescent probes in a myocyte, immobilized and spread on a treated with poly-L-lysine glass surface: red color, MitoTracker Orange CM- $\mathrm{H}_{2}$ TMRos (200 nM); green, DAF-FM $(10 \mu \mathrm{M})$; blue, specific to the nucleus dye Hoechst $33342(50 \mathrm{nM})$. The computer analysis of the fluorescent dyes distribution profiles (right panels). Data of two different experiments (top and bottom images). (See online version for color figure.) response of the probe in a wide range of concentrations from 0.1 to $10 \mu \mathrm{M}$ has found that $0.5 \mu \mathrm{M}$ is the optimal dye concentration to test the formation of $\mathrm{NO}$ in the presence of $\mathrm{Ca}^{2+}$ in the reaction medium. The fluorescence dependence on the concentration has a bell-shaped form, and $10 \mu \mathrm{M}$ DAF-FM fluorescence response showed a decrease in the signal below the control values (Fig. 2A). The time dependence of fluorescence has the appearance of a curve with the plateau (Fig. 2B), and the signal was significantly higher in the presence of non-mitochondrial $\mathrm{Ca}^{2+}(1 \mathrm{mM})$ in all cases, (Fig. 2), except for experiments with $10 \mu \mathrm{M}$ DAF-FM.
Data on the study of the isolated mitochondria NOsynthase activity depending on the presence of exogenous substrate and cofactors is presented in Fig. 3. The mitochondria were able to endogenous NO production in the working solution containing the substrates of respiration, namely $5 \mathrm{mM}$ pyruvate and succinate, and the balanced ionic composition. The biosynthesis of NO was found to be lower by $40 \%$ in the absence of respiration substrates. Sequential addition of $1 \mu \mathrm{M} \mathrm{L}$-arginine and $100 \mu \mathrm{M} \mathrm{Ca}{ }^{2+}$ into the incubation medium resulted in a significant $(p<0.05)$ increase in NO biosynthesis regarding to the control values. The addition
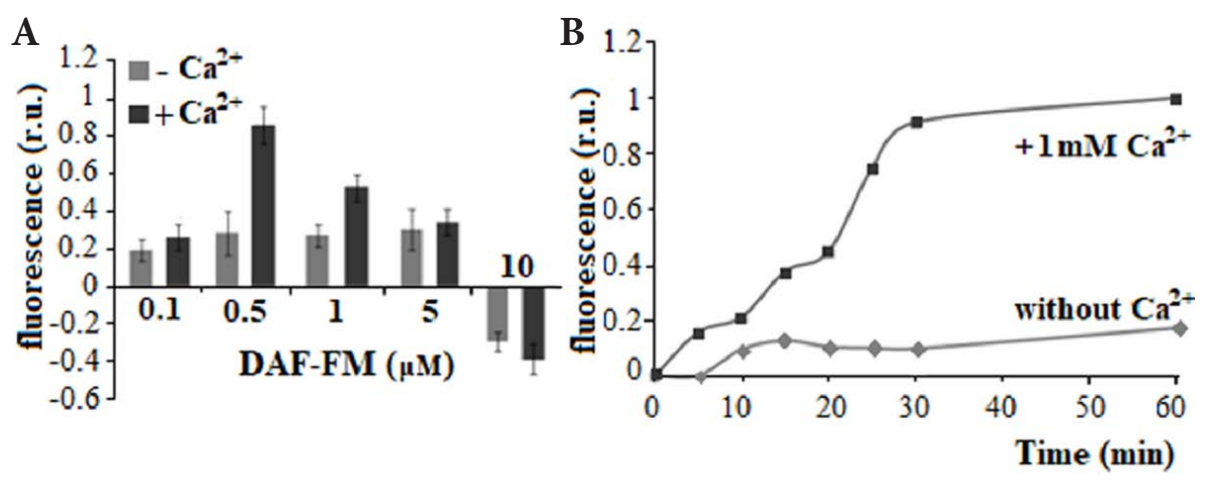

Figure 2. Dependency of DAF-FM fluorescence in isolated mitochondria on probe concentration (A) and on incubation time (B). Data are means \pm SE, $n=5$. B. Typical experiment of changes in fluorescent intensity of DAF-FM in time. r.u., relative units. 


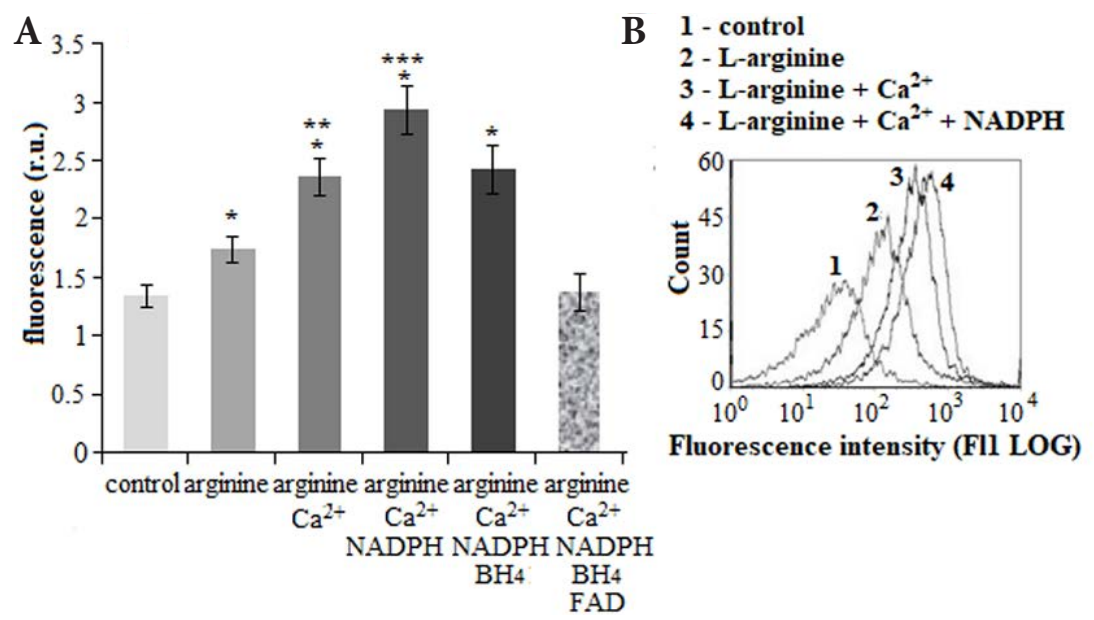

Figure 3. A. Dependency of mtNOS activity on the presence of substrate $(1 \mu \mathrm{M}$ L-arginine), $10 \mu \mathrm{M} \mathrm{NADPH}$ and cofactors $\left(100 \mu \mathrm{M} \mathrm{Ca}^{2+}, 10 \mu \mathrm{M} \mathrm{BH}_{4}, 10 \mu \mathrm{M}\right.$ FAD $)$ in mitochondria. Data are means $\pm \mathrm{SE}, n=7$. ${ }^{*}$ $p<0.05 v$ s. control, ${ }^{* *} p<0.05 v s$. arginine, ${ }^{* * *} p<0.05 v s$. arginine $+\mathrm{Ca}^{2+}$. B. Typical experiment of the displacement of the fluorescence intensity peaks in conditions of different medium composition. The time of the mitochondria incubation with substances was 30 minutes. r.u., relative units.

of exogenous $\mathrm{Ca}^{2+}$ revealed a significant increase $(p<0.05)$ of NO formation in relation to the experiments with its absence (Fig. 3). Adding $10 \mu \mathrm{M}$ of NADPH has also resulted in further growth of NO-synthase activity. At the same time, the introduction of $\mathrm{BH}_{4}$ into the reaction medium did not stimulated NO-synthase activity of mitochondria (Fig. 3). The presence of $10 \mu \mathrm{M}$ FAD resulted in a significant reduction in NO formation in the incubation medium containing all of the above-mentioned components. The concentration of the substances was used considering the results of such experiments on other objects.

The perforation of the internal mitochondrial membrane with a channel forming antibiotic alamethicin $(7.5 \mu \mathrm{g} / \mathrm{ml})$ did not significantly affected NO-synthase activity of the mitochondria in the presence of the substrate and cofactors in the reaction medium (graphic data is not given).

The dependence of NO-synthase activity of isolated mitochondria on $\mathrm{Ca}^{2+}$ and L-arginine concentrations in the incubation medium has been studied. As the concentration of exogenous $\mathrm{Ca}^{2+}$ increased, $\mathrm{NO}$ production by mitochondria augmented. The maximum of NO-synthase activity was observed in the range from 100 to $250 \mu \mathrm{M} \mathrm{Ca}^{2+}$ (Fig. 4). Further growth of $\mathrm{Ca}^{2+}$ concentration slightly reduced $\mathrm{NO}$ production. Concentration-dependent curve on Fig. 4 allows to calculate the activation constant of $\mathrm{NO}$-synthase reaction for $\mathrm{Ca}^{2+}$, which is $44.4 \pm 14.5 \mu \mathrm{M}(n=5)$ at $1 \mu \mathrm{M} \mathrm{L}$-arginine in the incubation medium.

It has also been established that the growth of NO biosynthesis with a tendency to enter the plateau level (the concentration of exogenous $\mathrm{Ca}^{2+}$ is $100 \mu \mathrm{M}$ ) is observed with increasing concentration of L-arginine from 1 to $75 \mu \mathrm{M}$, which allows us to calculate the apparent affinity constant for the substrate. NO formation is significantly reduced by further increase in the concentration of amino acid (up to $100 \mu \mathrm{M}$ in these experiments) (Fig. 5). The apparent affinity constant for $\mathrm{L}$-arginine is $28.9 \pm 9.1 \mu \mathrm{M}$ being calculated in Heinz coordinates $(n=5)$.

A widespread inhibitor of the constitutive NO-synthase NA in micromolar concentrations inhibited NO synthesis in mitochondria of myometrium (Fig. 6). A known blocker of $\mathrm{Ca}^{2+}$-transport in the internal membrane of mitochondria RuR $(10 \mu \mathrm{M})$ significantly inhibited NO production by mi-
A

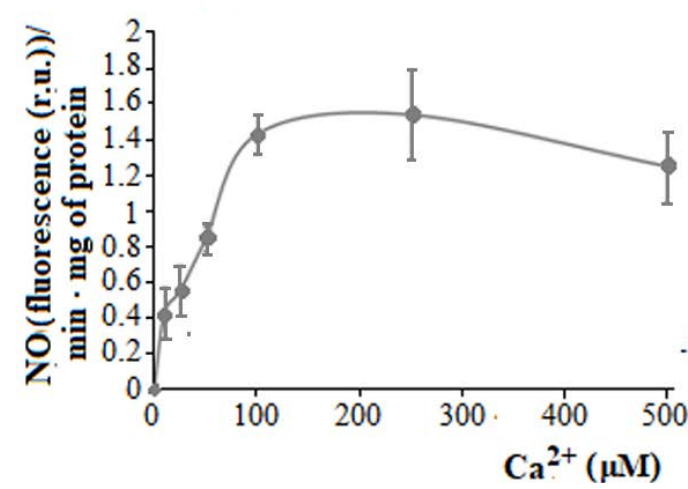

B

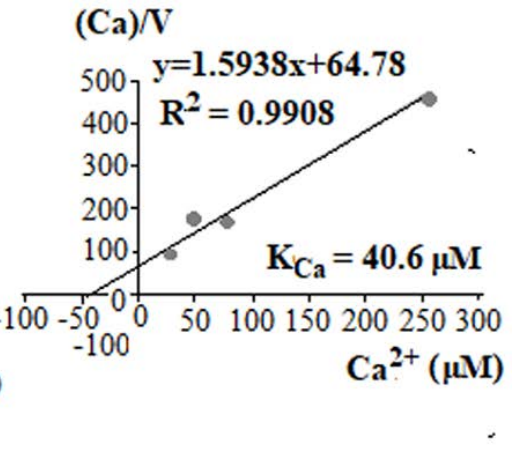

Figure 4. A. Dependency of mtNOS activity on exogenous $\mathrm{Ca}^{2+}$ concentration, data are means \pm SE, $n=5$. B. Example of activation constant calculation by Heinz method. r.u., relative units; $\mathrm{V}$, mtNOS activity. 


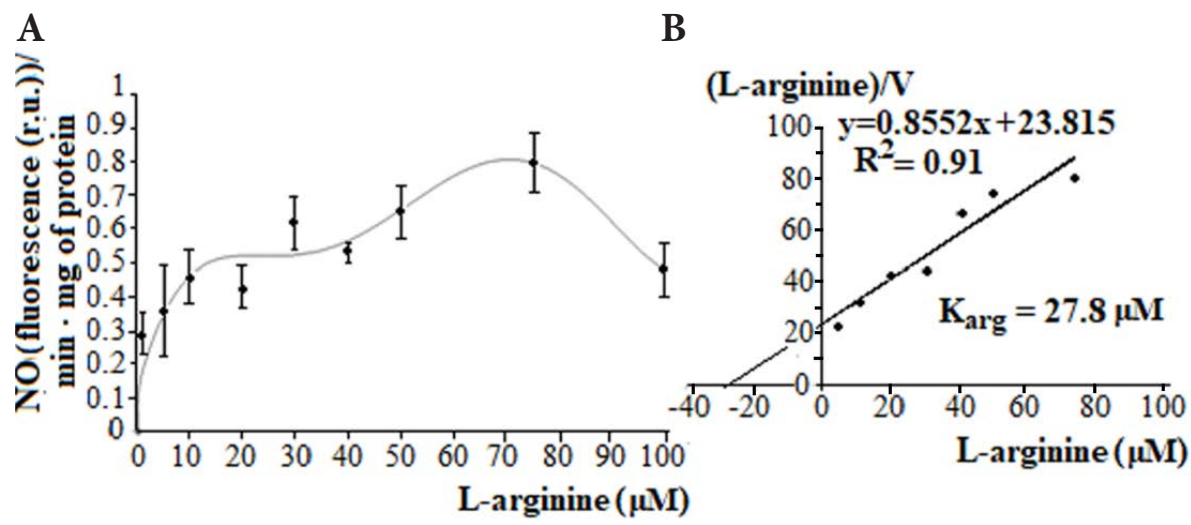

Figure 5. A. Dependency of mtNOS activity on exogenous L-arginine concentration. Data are means \pm SE, $n=5$. B. Example of apparent affinity constant for L-arginine calculation by Heinz method. r.u., relative units; $\mathrm{V}$, mtNOS activity. tochondria in the presence of $100 \mu \mathrm{M} \mathrm{Ca}^{2+}$ in the incubation medium (Fig. 6).

The dependence of NO-synthase activity of mitochondria on their energy status has been also studied. Isolated mitochondria synthesized NO in the absence of exogenously added substrates (Fig. 7), although with insignificant efficiency. The addition of $5 \mathrm{mM}$ pyruvate and succinate in the incubation medium caused the stimulation of their oxidation and the functioning of the electron transport chain was enhanced. Mitochondria were energized and the NO synthesis was increased. The similar effect on NO-synthase activity was carried out with the widespread inhibitor of $\mathrm{H}^{+}$-ATPase $\mathrm{H}^{+}$channel ( $\mathrm{F}_{\mathrm{O}}$ complex) oligomycin $(2.5 \mu \mathrm{M})$. The addition of $0.5 \mathrm{mM}$ ADP (inorganic phosphate and $\mathrm{Mg}^{2+}$ were present in the incubation medium) to the energized mitochondria resulted in the creation of conditions for ATP synthesis, and weakening of NO-synthase activity in mitochondria (Fig. 7). The blocking of the electron transport chain complex I with rotenone $(5 \mu \mathrm{M})$ or complex III with antimycin A $(1 \mu \mathrm{g} /$ $\mathrm{ml}$ ) resulted in a significant decrease in NO biosynthesis in energized mitochondria (Fig. 7). The similar effect was caused by CCCP, added into the incubation medium, which rapidly destroyed the electrochemical gradient of protons on the internal mitochondrial membrane (Fig. 7).

\section{Discussion}

The presence of NO in uterine smooth muscle cells, in particular in mitochondria, has been visualized, involving
A

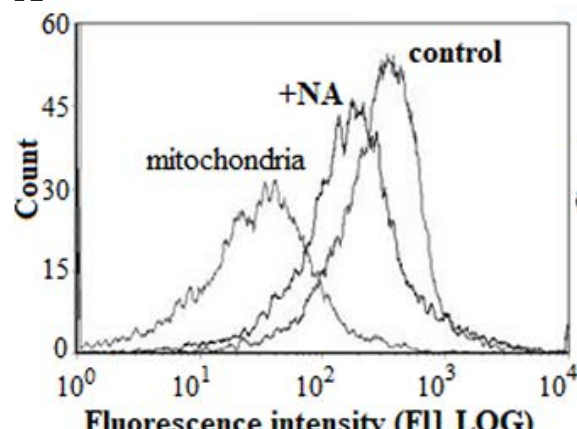

Fluorescence intensity (FIl LOG)

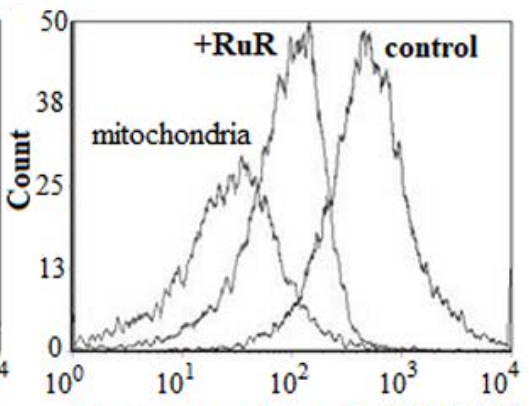

Fluorescence intensity (FIl LOG)

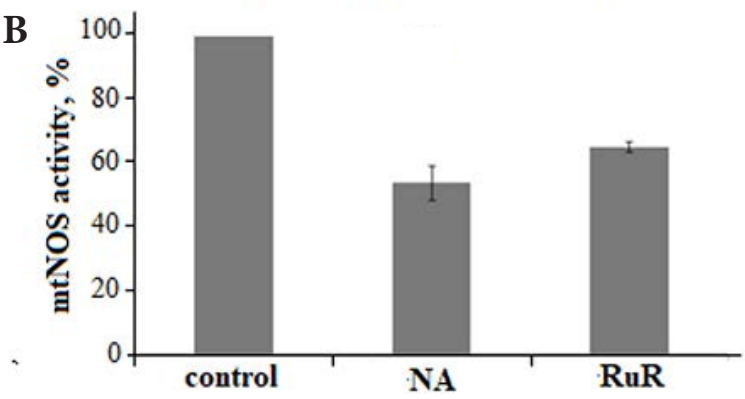

Figure 6. A. Typical experiment of the displacement of the fluorescence intensity peaks in condition of $\mathrm{N}^{\mathrm{G}}$-nitro-L-arginine (NA) and ruthenium red (RuR) action. Mitochondria, peak position at " 0 " time; control, peak position after $30 \mathrm{~min}$. B. Changes in fluorescent intensity of DAF-FM in mitochondria after adding $25 \mu \mathrm{MNA}$ and $10 \mu \mathrm{M}$ RuR. Data are means \pm SE, $n=5$. 


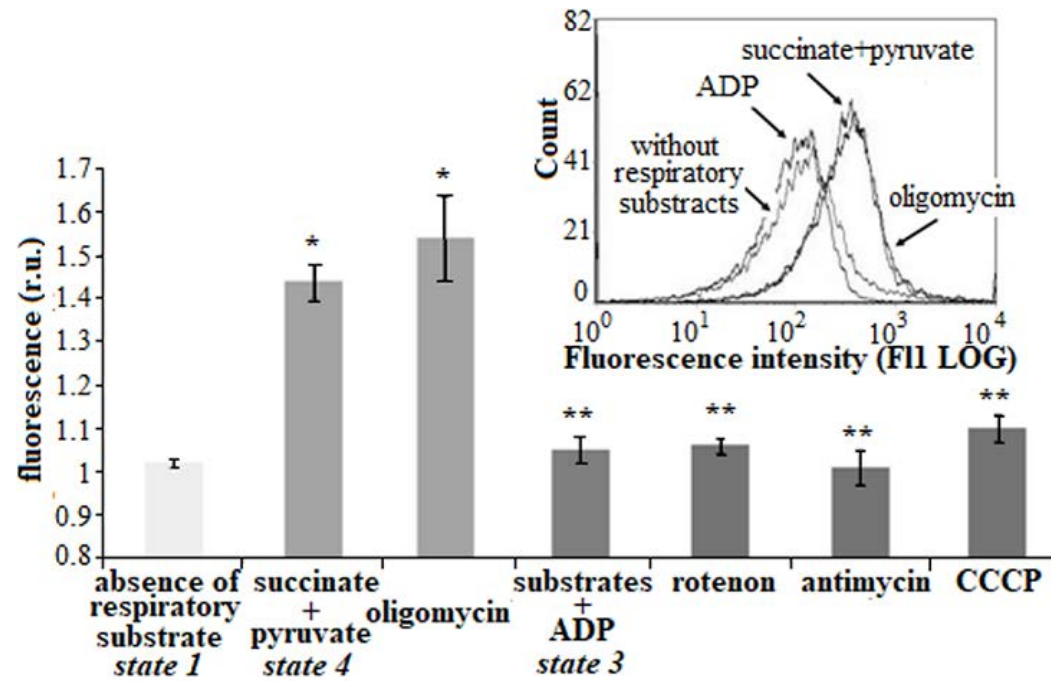

Figure 7. Dependency of mtNOS activity on the presence of respiratory substrates and electron transport chain inhibitors in mitochondria: $5 \mathrm{mM}$ pyruvate, $5 \mathrm{mM}$ succinate, $2.5 \mu \mathrm{M}$ oligomycin, $0.5 \mathrm{mM}$ ADP, $5 \mu \mathrm{M}$ of rotenone, $1 \mu \mathrm{g} / \mathrm{ml}$ of antimycin $\mathrm{A}, 10 \mu \mathrm{M}$ protonophore CCCP. States 1, 3, 4 , mitochondria respiration states by Chance and Williams (1955). ${ }^{*} p<0.05 v$ s. state $1,{ }^{* *} p<0.05$ vs. state 4 . Data are means $\pm \mathrm{SE}, n=5$. Insert: the fluorescence intensity peaks displacement in condition of different medium composition; the result of a typical experiment. r.u., relative units. fluorescence confocal microscopy, NO-sensitive DAF-FM probe and the specific marker of functionally active (energized) mitochondria MitoTracker Orange CM-H2TMRos. The use of DAF-FM fluorescent dye in these experiments is logical, since this probe is less sensitive to the action of laser and does not photo-bleach with time (Li and Wan 2015). Computer analysis of cell slices, that was chosen outside the cell nucleus (red lines on Fig. 1), showed identical profile of distribution of both fluorescent dyes. The co-localization of these dyes in freshly-isolated rat uterus myocytes has been observed, which indicates the possibility of NO formation in mitochondria of the myometrium (Fig. 1).

Subsequent studies have been conducted on isolated mitochondria of the rat myometrium by using DAF-FM and the flow cytometry method. According to the preliminary results, isolated mitochondria are oval particles with a size of 500-700 nm and cristae (own data of electron microscopy and photon correlation spectroscopy) (Kolomiets et al. 2013). These sub-cell structures had an intact inner membrane, their hydrodynamic diameter increased with adding a channel forming antibiotic alamethicin. Freshly-isolated mitochondria were able to accumulate $\mathrm{Ca}$ ions from the incubation medium in the presence of respiratory substrates and $\mathrm{Mg}$-ATP ${ }^{2-}$ (Kolomiets et al. 2013). Consequently, they have certain morphological and functional characteristics of intact organelles.

We have selected optimal concentrations of fluorescent dye to determine changes in NO content in the mitochondrial suspension using flow cytometry. This method has significant advantages over conventional spectrofluorimetry. They include a high signal/noise ratio in the fluorescence channel, an estimate of the fluorescence change of mitochondria precisely rather than the working medium and non-mitochondrial subcellular fragments, as well as the possibility of using a relatively small number of membrane preparations in the samples. It has been found that the optimal concentration of DAF-FM is $0.5 \mu \mathrm{M}$ in the presence of exogenous $\mathrm{Ca}^{2+}$. Both lower and higher concentrations of the probe turned out to be less effective. There was even a decrease in fluorescence referring to control values for $10 \mu \mathrm{M}$ DAF-FM, which is probably due to the interaction between the probe molecules (Fig. 2A). In the study of time dependence of enzymatic activity it has been established that the steady state is reached up to 30 minutes (Fig. 2B), which we have used for further study of NO biosynthesis by mitochondria.

mtNOS, like non-mitochondrial NOS isoforms, requires L-arginine, $\mathrm{O}_{2}, \mathrm{Ca}^{2+}$ as well as NADPH and cofactors (calmodulin, $\mathrm{BH}_{4}$ ) for the synthesis of NO. It is predicted, that mtNOS is co-localized together with the complex I of the respiratory chain at least in the case of heart mitochondria (Parihar et al. 2008; Zaobornyj and Ghafourifar 2012). Such specific compartmentalization involves the possibility of regulation of NOS activity by the local microenvironment of the organelles and the components of the matrix, as well as the dependence of NO production on the activity of the electron transport chain and the electrochemical gradient of hydrogen ions on the internal mitochondrial membrane. We tried to find out the necessity of certain components for constitutive NO-synthase reactions in the case of isolated myometrium mitochondria mtNOS. It should be noted that isolated mitochondria possess basal NO-synthase activity in the absence of cofactors and substrate, indicating the presence of necessary reaction components in the matrix in sufficient quantities and the energetic state of mitochondria in the medium with pyruvate and succinate (Fig. 3). The synthesis of NO was significantly lower in non-energized mitochondria without exogenous respiratory substrates 
(see comments on Fig. 7). Addition of $1 \mu \mathrm{M}$ L-arginine to the mitochondria significantly increased NO production regarding the control values. The effect of L-arginine in these concentrations can be surprising. It's the fact that the concentration of this amino acid in the matrix of liver mitochondria reaches a level of $150-300 \mu \mathrm{M}$, according to the experimental data (it is supposed that the level in cardiomyocytes is lower) (Giulivi et al. 1998). It is difficult to assume that NOS is in short supply, since its affinity for L-arginine is in the micromolar range. However, the well-known phenomenon is the so-called "arginine paradox", which is based on the fact that the introduction of L-arginine into the body is accompanied by an increase in the biosynthesis of $\mathrm{NO}$ (the concentration of this amino acid in liquids and tissues reaches millimoles) (Elms et al. 2013). It is believed that the presence in cells of asymmetric dimethylarginine, which competes with L-arginine, plays an essential role in this phenomenon (Palm et al. 2007; Peng et al. 2016). It should also be taken into account that the presence and high activity of arginase II in mitochondria cleaves the amino acid (Morris 2009).

Addition of $100 \mu \mathrm{M} \mathrm{Ca}^{2+}$ to the working medium resulted in further significant growth of NO production by mitochondria (Fig. 3) in relation to the L-arginine values. It is known that energized mitochondria of the heart synthesize NO even in the absence of exogenous $\mathrm{Ca}^{2+}$ due to the sufficient concentration of $\mathrm{Ca}^{2+}$ in the matrix (Elfering et al. 2002; Zaobornyj and Ghafourifar 2012). NO biosynthesis, which is independent from exogenous $\mathrm{Ca}^{2+}$ too, is observed in our experiments. However, $\mathrm{Ca}^{2+}$ addition to mouse and rat heart mitochondria leads to an increase in NO production, and extra-mitochondrial $\mathrm{Mg}^{2+}$, as well as RuR, suppresses it (Zaobornyj and Ghafourifar 2012). Thus, the biosynthesis of NO by mitochondria of myometrium also depends on the concentration of exogenous $\mathrm{Ca}^{2+}$.

The introduction of $10 \mu \mathrm{M}$ NADPH as the main source of electrons for non-mitochondrial NOS into the incubation medium in the presence of $100 \mu \mathrm{M} \mathrm{Ca}^{2+}$ and $1 \mu \mathrm{M} \mathrm{L}$-arginine resulted in further growth of $\mathrm{NO}$ production (Fig. 3). On the other hand, addition of $\mathrm{BH}_{4}(10 \mu \mathrm{M})$, a cofactor and a dissociation carrier of electrons, did not caused any probable difference in the biosynthesis of NO concerning the results in the absence of the cofactor (Fig. 3). Perhaps these results can be explained by the sufficient mitochondria energization in the conditions of our experiments (presence of succinate and pyruvate as respiratory substrates in the medium), functional coupling of mtNOS with the respiratory chain, as well as the possibility of reverse transport of electrons from II to I complex. In the case of $\mathrm{L}$-arginine or $\mathrm{BH}_{4}$ lack and stimulation of $\mathrm{Ca}^{2+}$ accumulation by mitochondria, $\mathrm{NO}$ formation was significantly lower, but at the same time there was an increase in the production of active forms of oxygen (Dedkova and Blatter 2009). Thus, according to cited data, the presence of $\mathrm{BH}_{4}$ in the incubation medium is a prereq- uisite for the correct testing of NO-synthase activity. This was taken into account for further experiments.

Addition of $10 \mu \mathrm{M}$ FAD as an essential element of electron transport from NADPH to the heme prosthetic group of nonmitochondrial NOS has a significant inhibitory effect on NO production by mitochondria (Fig. 3). We explain this effect by the specific location and, accordingly, the functioning of mtNOS as a part of complex I or IV of the respiratory chain. Due to the presence of a sufficient amount of calmodulin in mitochondria, this cofactor was absent in the medium of incubation. It should be noted that calmodulin antagonists, namely, trifluoperazine and calmidazolium, significantly inhibited NO-synthase activity in myometrium mitochondria (our unpublished data).

In all separate variants of the above-described experiments we used alamethicin $(7.5 \mu \mathrm{g} / \mathrm{ml})$ as an agent that forms nonspecific permeability of the internal mitochondrial membrane to cofactors and substrates (Gostimskaya et al. 2003). After alamethicin insertion, the value of the membrane potential decreases to the Donnan potential in isolated mitochondria (Nicholls and Ferguson 2002). Although alamethicin may increase the availability of exogenous cofactors and substrates and mtNOS activity may increase, mitochondrial depolarization will have the opposite effect. We have not registered any significant effect of alamethicin on NO products.

There is evidence that energized mitochondria are capable to produce $\mathrm{NO}$ in the absence of NADPH, L-arginine and exogenous $\mathrm{Ca}^{2+}$ in the incubation medium (Elfering et al. 2002; Valdez et al. 2006; Zaobornyj and Ghafourifar 2012). The intra-mitochondrial concentration of NADPH is 1.04$1.78 \mathrm{mM}$, which significantly exceeds the corresponding value of $\mathrm{K}_{\mathrm{NADPH}}$ for mtNOS $(0.1-1 \mu \mathrm{M})$ (Valdez et al. 2006). The concentration of L-arginine in the mitochondrial matrix corresponds to $150-310 \mu \mathrm{M}$ and is substantially higher than the $\mathrm{K}_{\mathrm{m}}$ for L-arginine ( $5 \mu \mathrm{M}$ for liver mitochondria (Giulivi et al. 1998), $36 \mu \mathrm{M}$ for cardiomyocytes (Alvarez and Boveris 2004). Thus, the concentrations of $\mathrm{L}$-arginine, $\mathrm{Ca}^{2+}$ and $\mathrm{NADPH}$ are not rate-limiting $\mathrm{NO}$ synthesis factors in mitochondria (Giulivi 1998; Zaobornyj and Ghafourifar 2012; Porcelli et al. 2014). Lack of L-arginine in the matrix and the corresponding decrease in mtNOS activity may be due to the increased activity of arginase II in mitochondria, which competes with the NOS for the substrate (Morris 2009).

Consequently, NO-synthase activity in isolated myometrium mitochondria has been identified in the presence of appropriate cofactors and substrates in the reaction medium using DAF-FM fluorescence probe and the flow cytometry method. The analysis of the results suggests that the optimal work of mtNOS in myometrium mitochondria requires the presence of respiration substrates, L-arginine, $\mathrm{Ca}^{2+}$ and NADPH. These data will be used for further studies of kinetic and catalytic properties of NO biosynthesis in mitochondria. 
Dependence of enzymatic activity on the concentration of exogenous $\mathrm{Ca}^{2+}$ in the range of $10-500 \mu \mathrm{M}$ has been investigated (Fig. 4). It has been found that the optimal range of concentrations of $\mathrm{Ca}^{2+}$ is $100-250 \mu \mathrm{M}$, further increase in the content of $\mathrm{Ca}^{2+}$ decreased enzymatic activity. The activation constant for $\mathrm{Ca}^{2+}$ is about $45 \mu \mathrm{M}$. This concentration of $\mathrm{Ca}^{2+}$ is close to the physiological value near mitochondria, where $\mathrm{Ca}^{2+} /$ inositol-1,4,5-trisphosphate $\left(\mathrm{IP}_{3}\right)$-induced $\mathrm{Ca}^{2+}$ release from the sarco(endo)plasmatic reticulum creates significant local $\mathrm{Ca}^{2+}$ concentrations (Graier et al. 2007). For comparison, the activation constant for energy-dependent accumulation of $\mathrm{Ca}^{2+}$ by mitochondria reaches $54 \mu \mathrm{M}$ (according to our preliminary calculations) (Kolomiets et al. 2013). E. Dedkova and L. Blatter showed a dose-dependent increase in $\mathrm{NO}$ production in mitochondria in the presence of $\mathrm{L}$-arginine and an increase in the concentration of $\mathrm{Ca}^{2+}$ in myocytes permeabilized by digitonin (Dedkova and Blatter 2009). In this work NO-sensitive fluorescence probe 4,5-diaminofluorescein diacetate (DAF-2) and MitoTracker Red CMXRos mitochondrial specific dye were used.

The production of NO was analyzed according to the changes in L-arginine concentration in the range of 1-100 $\mu \mathrm{M}$ (Fig. 5). It has been established that the dependence of enzymatic activity on the concentration of the substrate has a bell-shaped character, and the apparent affinity constant for $\mathrm{L}$-arginine is about $30 \mu \mathrm{M}$. For comparison, the corresponding constants for mitochondria of the heart make up 35-36 $\mu \mathrm{M}$, the skeletal muscle (diaphragm) - $37 \mu \mathrm{M}$, and the liver - $5 \mu \mathrm{M}$ (Tatoyan and Giulivi 1998; Alvares and Boveris 2004). The authors who have carried out these calculations emphasize that the apparent affinity constants are several times lower than the calculated concentration of L-arginine in the matrix.

A nonspecific inhibitor of the constitutive NOS NA at a concentration of $25 \mu \mathrm{M}$ inhibited NO production in mitochondria almost by $50 \%$ (Fig. 6). This fact suggests that mtNOS is one of the variants of constitutive enzymes, possibly neuronal NOS. These isoforms are $\mathrm{Ca}^{2+}$. dependent, therefore the biosynthesis of $\mathrm{NO}$ is suppressed by ruthenium red, a known blocker of $\mathrm{Ca}^{2+}$ transport in mitochondria (Fig. 6). However, there is evidence that the outer membrane of mitochondria of endothelial cells contains endothelial NOS, and $85 \%$ of the mitochondria of the heart are positive in relation to this isoform. Solubilized and purified by affinity chromatography, liver mtNOS synthesized $250-350 \mathrm{nmol} \mathrm{NO} / \mathrm{min} / \mathrm{mg}$ protein, the enzyme was inhibited by L-NMMA. A detailed analysis of the amino acid composition of mtNOS found that the protein structure corresponds to the initial sequence of nNOS (Tatoyan and Giulivi 1998).

Thus, important kinetic parameters of NO-synthase activity in mitochondria are calculated: the apparent affinity constant for L-arginine and the activation constant for
$\mathrm{Ca}^{2+}$, which have a physiological meaning. NO synthesis was inhibited by a known inhibitor of constitutive NOS NA and was dependent on $\mathrm{Ca}^{2+}$ concentration.

The current conception of mitochondria metabolic states and respiratory control is based on the rate of $\mathrm{O}_{2}$ uptake by isolated organelles. The high rate of respiration and ATP production have been observed in metabolic state 3 in case of addition of respiratory substrates and ADP. The absence of ADP causes low rate of $\mathrm{O}_{2}$ uptake, ATP is not synthesized, and the internal mitochondrial membrane is hyperpolarized (metabolic state 4) (Chance and Williams 1955; Valdez et al. 2006). We obtained the following results, using the panel of the electron transport chain modifiers. Isolated mitochondria produced NO in the absence of exogenously added substrates of respiration at the internal reserves. This process in conditions of mitochondria energization and the hyperpolarization of their internal membrane is significantly enhanced by addition of respiratory substrates or oligomycin (Fig. 7). Instead, when effective oxidative phosphorylation occurs (state 3 ), the synthesis of NO decreases. The inhibition of certain complexes of the respiratory chain by rotenone and antimycin, as well as the dissipation of the electrochemical gradient by protonophore CCCP, suppresses mtNOS (Fig. 7). The transition from relative rest (state 4 ) to active respiration in case of fully coupled mitochondria (available substrates of respiration, sufficient oxygen concentration and present ADP, state 3 ) is accompanied by an decrease in NO production in heart mitochondria. NO release exponentially depends on the value of the inner membrane electric potential (Valdez 2006). Formation of NO is observed even without exogenous NADPH under the condition of electron transport from II to I complex in the presence of ATP and succinate. mtNOS activity is maximal during optimal functionality of the electron transport chain, in particular, complex I, which is the source of electrons for the functioning of the enzyme. Inactivation of complex I reduces mtNOS activity (Parihar et al. 2008).

Our results are consistent with those obtained on permeabilized cardiomyocytes using the method of confocal microscopy. In particular, collapse of the membrane potential in the presence of protonophore FCCP, blockage of the $\mathrm{Ca}^{2+}$-uniporter of the internal membrane of mitochondria with the compound Ru360, the use of the electron transport chain inhibitors rotenone or antimycin A in combination with oligomycin resulted in inhibition of NO synthesis in mitochondria (Dedkova and Blatter 2009).

It should be noted that the decrease in NO production during the transition from the state 4 to 3 is the opposite of what one could expect from the slight matrix acidification and $\mathrm{pH}$ dependence of mtNOS activity. Dependence of NO products on mitochondrial $\mathrm{pH}$ in the range of 5.5-8.5 has a bell-shaped character with a maximum at $\mathrm{pH} 7.4$ (Valdez et al. 2006). Taking into account the $\mathrm{pH}$ value of the matrix 
at about 7.5 in the state 3 and about 7.8 in the state 4 , mtNOS activity should be higher in the first case. These results indicate that a larger synthesis of NO in the state 4 is not associated with the changes in $\mathrm{pH}$. There is a view that mtNOS activity is regulated by an electrical, not a proton component of the membrane potential on the internal mitochondrial membrane (Valdez et al. 2006).

Thus, our results convincingly show and are in line with the notion that the biosynthesis of NO by mitochondria significantly depends on the level of their energy and the magnitude of hydrogen ion electrochemical gradient on the inner membrane.

\section{Conclusions}

The formation of $\mathrm{NO}$ in mitochondria of uterine smooth muscle cells has been firstly demonstrated in the presented work using laser confocal microscopy and the methodology of the specific fluorescent dyes co-localization. The conditions for determining NO-synthase activity in isolated mitochondria of rat myometrium have been selected employing DAF-FM fluorescence probe and flow cytometry. The optimal work of mtNOS in isolated myometrium mitochondria requires the presence of respiration substrates, $\mathrm{Ca}^{2+}$, NADPH and L-arginine. The key kinetic parameters of this enzymatic reaction are calculated: the apparent affinity constant for L-arginine is $28.9 \pm 9.1 \mu \mathrm{M}$, and the activation constant for $\mathrm{Ca}^{2+}$ is $44.4 \pm 14.5 \mu \mathrm{M}$, which has a physiological value. It is proved that the biosynthesis of NO by mitochondria significantly depends on the level of their energization, the intensity of exogenous $\mathrm{Ca}^{2+}$ entry to the matrix and suppression by $\mathrm{N}^{\mathrm{G}}$-nitro-L-arginine.

Conflict of interest. Authors declare no conflict of interest.

Acknowledgement and financial support. Authors received no grant for this study. Authors thank Dr. Sergiy O. Karakhim and Dr. Alexander Y. Chunikhin for their technical assistance.

\section{References}

Alvarez S, Boveris A (2004): Mitochondrial nitric oxide metabolism in rat muscle during endotoxemia. Free Radic Biol. Med. 37, $1472-1478$

https://doi.org/10.1016/j.freeradbiomed.2004.06.034

Boveris A, Valdez LB, Alvarez S, Zaobornyj T, Boveris AD, Navarro A (2003): Kidney mitochondrial nitric oxide synthase. Antioxid. Redox Signal. 5, 265-271 https://doi.org/10.1089/152308603322110841

Boveris A (1977): Mitochondrial production of superoxide radical and hydrogen peroxide. In: Tissue Hypoxia and Ischemia (Eds. M Reivich, R Coburn, S Lahiri and B Chance), pp. 67-82, Plenun Publishing corporation, NewYork https://doi.org/10.1007/978-1-4615-9035-4_5

Bradford MM (1976): A rapid and sensitive method for the quantitation of microgram quantities of protein utilizing the principle of protein-dye binding. Anal. Biochem. 72, 248-254 https://doi.org/10.1016/0003-2697(76)90527-3

Brown GC (2007): Nitric oxide and mitochondria. Front Biosci. 12, 1024-1033 https://doi.org/10.2741/2122

Brown GC, Borutaite V (2007): Nitric oxide and mitochondrial respiration in the heart. Cardiovasc. Res. 75, 283-290 https://doi.org/10.1016/j.cardiores.2007.03.022

Buckman JF, Hernandes H, Kress GJ, Votyakova TV, Pal S, Reynolds IJ (2001): MitoTracker labeling in primary neuronal and astrocytic cultures: influence of mitochondrial membrane potential and oxidants. J. Neurosc. Meth. 104, 165-176 https://doi.org/10.1016/S0165-0270(00)00340-X

Bustamante J, Bersier G, Badin RA, Cymeryng C, Parodi A, Boveris A (2002): Sequential NO production by mitochondria and endoplasmic reticulum during induced apoptosis. Nitric Oxide 6, 333-341 https://doi.org/10.1006/niox.2001.0420

Buxton ILO (2004): Regulation of uterine function: a biochemical conundrum in the regulation of smooth muscle relaxation. Mol. Pharmacol. 65, 1051-1059 https://doi.org/10.1124/mol.65.5.1051

Carreras MC, Poderoso JJ (2007): Mitochondrial nitric oxide in the signaling of cell integrated responses. Am. J. Physiol. Cell. Physiol. 292, C1569-1580 https://doi.org/10.1152/ajpcell.00248.2006

Chance B, Williams GR (1955): Respiratory enzymes in oxidative phosphorylation. III. The steady state. J. Biol. Chem. 217, 409-427

Davidson SM, Duchen MR (2006): Effects of NO on mitochondrial function in cardiomyocytes: Pathophysiological relevance. Cardiovasc. Res. 71, 10-21 https://doi.org/10.1016/j.cardiores.2006.01.019

Dedkova EN, Blatter LA (2009): Characteristics and function of cardiac mitochondrial nitric oxide synthase. J. Physiol. 587, $851-872$ https://doi.org/10.1113/jphysiol.2008.165423

Dolińska M, Albrecht J (1998): L-arginine uptake in rat cerebral mitochondria. Neurochem. Int. 33, 233-236 https://doi.org/10.1016/S0197-0186(98)00020-5

Elfering SL, Sarkela ThM, Giulivi C (2002): Biochemistry of mitochondrial nitric-oxide synthase. J. Biol. Chem. 277, 38079-38086

https://doi.org/10.1074/jbc.M205256200

Elms S, Chen F, Wang Y, Qian J, Askari B, Yu Y, Pandey D, Iddings J, Caldwell RB, Fulton DJ (2013): Insights into the arginine paradox: evidence against the importance of subcellular location of arginase and eNOS. Am. J. Physiol. Heart Circ. Physiol. 305, H651- 666 https://doi.org/10.1152/ajpheart.00755.2012

Forstermann U, Sessa WC (2012): Nitric oxide synthases: regulation and function. Eur. Heart J. 33, 829-837 https://doi.org/10.1093/eurheartj/ehr304

Franco MC, Antico Arciuch VG, Peralta JG, Galli S, Levisman D, Lopez LM, Romorini L, Poderoso JJ, Carreras MC (2006): Hy- 
pothyroid phenotype is contributed by mitochondrial complex i inactivation due to translocated neuronal nitric-oxide synthase. J. Biol. Chem. 281, 4779-4786 https://doi.org/10.1074/jbc.M512080200

Gellerich FN, Gizatullina Z, Trumbeckaitec S, Nguyen HP, Pallas Th, Arandarcikaite O, Vielhaber S, Seppet E, Striggow F (2010): The regulation of OXPHOS by extramitochondrial calcium. Biochim. Biophys. Acta 1797, 1018-1027 https://doi.org/10.1016/j.bbabio.2010.02.005

Ghafourifar P, Richter Ch (1997): Nitric oxide synthase activity in mitochondria. FEBS Lett. 418, 291-296 https://doi.org/10.1016/S0014-5793(97)01397-5

Ghafourifar P, Cadenas E (2005): Mitochondrial nitric oxide synthase. Trends Pharmacol. Sci. 26, 190-195 https://doi.org/10.1016/j.tips.2005.02.005

Ghimire K, Altmann HM,. Straub AC, Isenberg JS (2017): Nitric oxide: what's new to NO? Am. J. Physiol. Cell. Physiol. 312, C254-262 https://doi.org/10.1152/ajpcell.00315.2016

Giulivi C (1998): Functional implications of nitric oxide produced by mitochondria in mitochondrial metabolism. Biochem. J. 332, 673-679 https://doi.org/10.1042/bj3320673

Giulivi C, Poderoso JJ, Boveris A (1998): Production of nitric oxide by mitochondria. J. Biol. Chem. 273, 11038-11043 https://doi.org/10.1074/jbc.273.18.11038

Giulivi C, Kato K, Cooper ChE (2006): Nitric oxide regulation of mitochondrial oxygen consumption I: cellular physiology. Am. J. Physiol. Cell. Physiol. 291, C1225-1231 https://doi.org/10.1152/ajpcell.00307.2006

Giulivi C (2007): Mitochondria as generators and targets of nitric oxide. Novartis Found Symp. 287, 92-104 https://doi.org/10.1002/9780470725207.ch7

Gostimskaya IS, Grivennikova VG, Zharova TV, Bakeeva LE, Vinogradov AD (2003): In situ assay of the intramitochondrial enzymes: use of alamethicin for permeabilization of mitochondria. Anal. Biochem. 313, 46-52 https://doi.org/10.1016/S0003-2697(02)00534-1

Graier WF, Frieden M, Malli R (2007): Mitochondria and Ca2+ signaling: old quests, new functions. Eur. J. Physiol. 455, 375-396 https://doi.org/10.1007/s00424-007-0296-1

Haynes V, Elfering SL, Squires RJ, Traaseth N, Solien J, Ettl1 A, Giulivi C (2003): Mitochondrial Nitric-oxide Synthase: Role in Pathophysiology. IUBMB Life 55, 599-603 https://doi.org/10.1080/15216540310001628681

Haynes V, Elfering S, Traaseth N, Giulivi C (2004): Mitochondrial Nitric-Oxide Synthase: Enzyme Expression, Characterization, and Regulation. J. Bioenerg. Biomembr. 36, 341-346 https://doi.org/10.1023/B:JOBB.0000041765.27145.08

Keleti T (1986): Basic enzyme kinetics. Akademiai Kiado, Budapest

Kolomiets OV, Danylovych YuV, Danylovych HV, Kosterin SO (2013): Ca2+ accumulation study in isolated smooth muscle mitochondria using Fluo-4 AM. Ukr. Biochem. J. 85, 30-39 https://doi.org/10.15407/ubj85.04.030

Korshunov SS, Skulachev VP, Starkov AA (1997): High protonic potential actuates a mechanism of production of reactive oxygen species in mitochondria. FEBS Lett. 416, 15-18
https://doi.org/10.1016/S0014-5793(97)01159-9

Levine AB, Punihaole D, Levine TB (2012): Characterization of the role of nitric oxide and its clinical applications. Cardiology $122,55-68$ https://doi.org/10.1159/000338150

Li H, Wan A (2015): Fluorescent probes for real-time measurement of nitric oxide in living cells. Analyst 140, 7129-7141 https://doi.org/10.1039/C5AN01628B

Litvinova L, Atochin DN, Fattakhov N, Vasilenko M, Zatolokin P, Kirienkova E (2015): Nitric oxide and mitochondria in metabolic syndrome. Front. Physiol. 6, 20 https://doi.org/10.3389/fphys.2015.00020

Mollard P, Mironneau J, Amedee T (1986): Electrophysiological characterization of single pregnant rat myometrial cells in short-term primary culture. Am. J. Physiol. 19, C47-54 https://doi.org/10.1152/ajpcell.1986.250.1.C47

Morris SM (2009): Recent advances in arginine metabolism: roles and regulation of the arginases. British J. Pharmacol. 157, 922-930 https://doi.org/10.1111/j.1476-5381.2009.00278.x

Nicholls DG, Ferguson SJ (2002): Bioenergetics 3. Academic Press, London

Nisoli E, Carruba MO (2006): Nitric oxide and mitochondrial biogenesis. J. Cell Sci. 119, 2856-2862

https://doi.org/10.1242/jcs.03062

Palm F, Onozato ML, Luo Z, Wilcox CS (2007): Dimethylarginine dimethylaminohydrolase (DDAH): expression, regulation, and function in the cardiovascular and renal systems. Am. J. Physiol. Heart. Circ. Physiol. 293, H3227- 3245 https://doi.org/10.1152/ajpheart.00998.2007

Parihar MS, Nazarewicz RR, Kincaid E, Bringold U, Ghafourifar P (2008): Association of mitochondrial nitric oxide synthase activity with respiratory chain complex I. Biochem. Biophys. Res. Commun. 366, 23-28 https://doi.org/10.1016/j.bbrc.2007.11.056

Peng H, Chen L, Huang X, Yang T, Yu Z, Cheng G, Zhang G, Shi R (2016): Vascular peroxidase 1 up regulation by angiotensin II attenuates nitric oxide production through increasing asymmetrical dimethylarginine in HUVECs. J. Am. Soc. Hypertens. 10, 741-751 https://doi.org/10.1016/j.jash.2016.06.036

Persichini T, Mazzone V, Polticelli F, Moreno S, Venturini G, Clementi E, Colasanti M (2005): Mitochondrial type I nitric oxide synthase physically interacts with cytochrome c oxidase. Neurosci. Lett. 384, 254-259 https://doi.org/10.1016/j.neulet.2005.04.085

Piantadosi CA, Suliman HB (2012): Redox regulation of mitochondrial biogenesis. Free Radic. Biol. Med. 53, 2043-2053 https://doi.org/10.1016/j.freeradbiomed.2012.09.014

Porcelli V, Fiermonte G, Longo A, Palmieri F (2014): The human gene SLC25A29, of solute carrier family $\mathbf{2 5}$, encodes a mitochondrial transporter of basic amino acids. J. Biol. Chem. 289, 13374-13384 https://doi.org/10.1074/jbc.M114.547448

Santos CXC, Anilkumar N, Zhang M, Brewer AC, Shah AM (2011): Redox signaling in cardiac myocytes. Free Radic. Biol. Med. 50, 777-793 https://doi.org/10.1016/j.freeradbiomed.2011.01.003 
Tatoyan A, Giulivi C (1998): Purification and characterization of a nitric-oxide synthase from rat liver mitochondria. J. Biol. Chem. 273, 11044-11048 https://doi.org/10.1074/jbc.273.18.11044

Tengan CH, Rodrigues GS, Godinho RO (2012): Nitric oxide in skeletal muscle: role on mitochondrial biogenesis and function. Int. J. Mol. Sci. 13, 17160-17184 https://doi.org/10.3390/ijms131217160

Traaseth N, Elfering S, Solien J, Haynes V, Giulivi C (2004): Role of calcium signaling in activation of mitochondrial nitric oxide synthase and citric acid cycle. Biochim. Biophys. Acta 1658, 64-71 https://doi.org/10.1016/j.bbabio.2004.04.015
Valdez LB, Zaobornyj T, Boveris A (2006): Mitochondrial metabolic states and membrane potential modulate mtNOS activity // Biochim. Biophys. Acta 1757, 166-172

https://doi.org/10.1016/j.bbabio.2006.02.013

Zaobornyj T, Ghafourifar P (2012): Strategic localization of heart mitochondrial NOS: a review of the evidence. Am. J. Physiol. Heart. Circ. Physiol. 303, H1283-1293

https://doi.org/10.1152/ajpheart.00674.2011

Received: May 29, 2018

Final version accepted: September 10, 2018

First published online: January 18, 2019 